\title{
TITLE:
}

\section{Decay of an oscillating plate in a free-molecular gas}

$\operatorname{AUTHOR}(\mathrm{S})$ :

Tsuji, Tetsuro; Aoki, Kazuo

CITATION:

Tsuji, Tetsuro ...[et al]. Decay of an oscillating plate in a free-molecular gas. AIP Conference Proceedings 2011, 1333: 140-145

\section{ISSUE DATE:}

2011

URL:

http://hdl.handle.net/2433/160666

\section{RIGHT:}

Copyright 2011 American Institute of Physics. This article may be downloaded for personal use only. Any other use requires prior permission of the author and the American Institute of Physics. The following article appeared in AIP Conference Proceedings 1333, 140 (2011) and may be found at http://link.aip.org/link/?apc/1333/140 


\title{
Decay of an oscillating plate in a free-molecular gas
}

\author{
Tetsuro Tsuji $i^{\dagger}$ and Kazuo Aoki ${ }^{\dagger}, \$$ \\ $\dagger$ Department of Mechanical Engineering and Science, Kyoto University, Kyoto 606-8501, Japan \\ $\doteqdot$ Advanced Research Institute of Fluid Science and Engineering, Kyoto University, Kyoto 606-8501, Japan
}

\begin{abstract}
An infinite plate without thickness is placed in a free-molecular gas, and an external force, obeying Hooke's law, is acting perpendicularly on the plate. If the plate is displaced perpendicularly from its equilibrium position and released, then it starts an oscillatory motion, which decays as time goes on because of the drag exerted by the gas molecules. This unsteady motion is investigated numerically, under the diffuse reflection condition, with special interest in the manner of its decay. It is shown that the decay of the displacement of the plate is slow and is in proportion to an inverse power of time. The result complements the existing mathematical study of a similar problem [S. Caprino, et al., Math. Models. Meth. Appl. Sci. 17, pp. 1369-1403 (2007)] in the case of non-oscillatory decay.
\end{abstract}

Keywords: Free-molecular gas, Kinetic theory of gases, Moving-boundary problems, Decay rate

PACS: 47.45.Dt, 47.45.Ab, 05.20.Dd, 51.10.+y

\section{INTRODUCTION}

Free-molecular gas (or Knudsen gas) is a highly rarefied gas in which collisions between gas molecules can be neglected completely. Since the free-molecular gas is, in general, much simpler and more tractable than a gas with intermolecular collisions, its properties have been studied extensively for steady problems (e.g. [1, 2, 3]). In timedependent problems, however, there arises a complexity that does not appear in a collisional gas. For instance, let us consider free unsteady motion of a body with acceleration or deceleration in a free-molecular gas. The gas molecules that are reflected by the body at early times may hit the body again at later times. In contrast to the collisional gas, such molecules transfer information about the body at an early stage directly to the body at a later stage and may affect the motion of the body long time later. In other words, they give rise to a sort of long-memory effect. In what follows, such multiple collisions of a gas molecule with the body will be called recollisions. The long-memory effect caused by the recolliding molecules in time-dependent problems of a free-molecular gas was already mentioned in [1]. However, to the best of the authors' knowledge, there had been no systematic study of this effect.

Recently, this effect was studied mathematically $[4,5,6,7]$ as well as numerically [8] in connection with the unsteady motion of a body in a free-molecular gas caused by a constant external force and its approach to the final steady motion. That is, it was shown that, because of the effect of recolliding molecules, the approach is slowed down and its rate becomes algebraic (i.e., proportional to an inverse power of time). To be more precise, if we let $t_{*}$ be the time variable, $V_{w}\left(t_{*}\right)$ the speed of the body, and $V_{w \infty}$ the final (constant) speed of the body, then it holds that

$$
\left|V_{w \infty}-V_{w}\left(t_{*}\right)\right| \approx C_{1} / t_{*}^{n}
$$

for sufficiently large $t_{*}$, where $C_{1}$ is a positive constant, and the positive integer $n$ depends on the dimension of the body as well as the type of gas-surface interaction.

In the present study, we consider the case of a variable external force that was studied mathematically in [5]. The problem considered in [5] is as follows. A disk of dimension $d$, placed in a free-molecular gas, is subject to an external force in the direction perpendicular to the disk. The external force obeys Hooke's law regarding the displacement of the disk from its equilibrium position (elastic force). Thus, if we denote by $X_{w}\left(t_{*}\right)$ the displacement of the disk in the direction opposite to the external force, then it is expressed as $-K X_{w}\left(t_{*}\right)$ with a positive constant $K$. The gas molecules are assumed to undergo specular reflection on the disk surface. Initially, the disk is fixed with displacement $X_{w 0}(>0)$, and the gas is in a uniform equilibrium state at rest. At time $t_{*}=0$, it is released with an initial velocity (parallel to the external force). Then, the disk, in general, starts oscillation, and as time goes on, the motion decays, i.e., $X_{w}\left(t_{*}\right) \rightarrow 0$ because of the drag exerted by the gas.

27th International Symposium on Rarefied Gas Dynamics, 2010

AIP Conf. Proc. 1333, 140-145 (2011); doi: 10.1063/1.3562639

(C) 2011 American Institute of Physics 978-0-7354-0888-0/\$30.00 
In [5], it was proved that there exist cases where $X_{w}\left(t_{*}\right)$ decays monotonically (without oscillation) and that the decay in such cases is described by

$$
\left|X_{w}\left(t_{*}\right)\right| \approx C_{2} / t_{*}^{d+2},
$$

with a positive constant $C_{2}$ for sufficiently large $t_{*}$. Our interest is whether the same decay rate is true or not in the case where the plate undergoes many oscillations before it stops. However, because of mathematical difficulty, analysis could not be extended to the case with oscillations in [5]. In the present study, therefore, we investigate this problem numerically. Since we need long-time computations with great accuracy to obtain an accurate decay rate, we restrict ourselves to the one-dimensional case (i.e., the disk is an infinite plate). In addition, we assume diffuse reflection, rather than specular reflection, on the surface of the plate. This facilitates computation for the same reason as in [8] (cf. Sec. V D of [8]).

It should be mentioned that the decay of oscillating motion of a pendulum in a Stokes fluid was studied in [9], in which the decay rate was shown to be proportional to $t_{*}^{-\gamma}(\gamma=1 / 2$ or $3 / 2)$.

\section{FORMULATION OF THE PROBLEM}

Let us consider an infinite plate without thickness immersed in an infinite expanse of an ideal monatomic gas in an equilibrium state at rest at temperature $T_{0 *}$ and density $\rho_{0 *}$. The temperature of the plate is uniform and kept at $T_{0 *}$. Suppose that the plate is placed at $X_{1}=X_{w 0},-\infty<X_{j}<\infty$ $(j=2,3)$, where $X_{i}$ is the Cartesian coordinate system, and that the plate is subject to an external force $F_{*}\left(t_{*}\right)$ in the $X_{1}$ direction per unit area of the plates (see Fig. 1). The force is assumed to obey Hooke's law (elastic force) and to be described by $F_{*}\left(t_{*}\right)=-M_{*} \omega_{*}^{2} X_{w}\left(t_{*}\right)$, where $M_{*}$ is the mass of the plate per unit area, and $\omega_{*}$ is the proper frequency of oscillation $\left(X_{1}=0\right.$ is the equilibrium position of the plate).

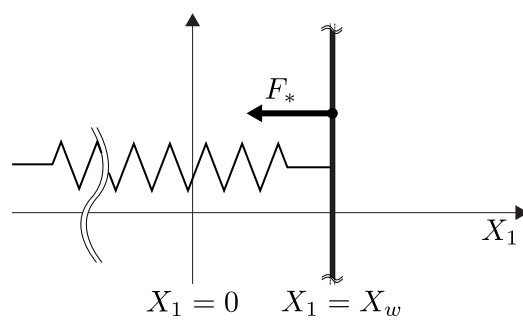

FIGURE 1. Configuration of the problem.

At time $t_{*}=0$, the plate is released with initial velocity $V_{w 0}$ (in the $X_{1}$ direction). We investigate the subsequent unsteady motion of the plate numerically with special interest in the decay of the motion and its rate, under the following assumptions: (i) The behavior of the gas is described by the Boltzmann equation; (ii) The gas is so rarefied that the collisions between the gas molecules can be neglected (free-molecular gas or Knudsen gas), and no external force acts on the gas molecules; and (iii) the gas molecules undergo diffuse reflection on the plate.

Before presenting the basic equations, we first introduce (and repeat) some necessary dimensional quantities: $X_{i}$ is the Cartesian coordinate system in space, $t_{*}$ the time variable, $\xi_{i}$ the molecular velocity, $X_{w}\left(t_{*}\right)$ the position of the plate ( $X_{1}$ coordinate), $V_{w}\left(t_{*}\right)$ the velocity of the plate (in the $X_{1}$ direction), $X_{w 0}$ the initial position of the plate, $V_{w 0}$ the initial velocity of the plate, $F_{*}\left(t_{*}\right)$ the external elastic force acting on the plate per unit area, $G_{*}\left(t_{*}\right)$ the drag acting on the plate per unit area, $M_{*}$ be the mass of the plate per unit area, and $f_{*}\left(X_{i}, \xi_{i}, t_{*}\right)$ the velocity distribution function of the gas molecules. Then, we introduce their dimensionless counterparts, $x_{i}, t, \zeta_{i}, x_{w}(t), v_{w}(t), x_{w 0}, v_{w 0}, F(t), G(t), M$, and $f\left(x_{i}, \zeta_{i}, t\right)$, and define them as

$$
\begin{array}{llll}
x_{i}=X_{i} / X_{\mathrm{ref}}, & t=t_{*} / t_{\mathrm{ref}}, & \zeta_{i}=\xi_{i} / c_{\mathrm{ref}}, & x_{w}(t)=X_{w}\left(t_{*}\right) / X_{\mathrm{ref}}, \\
v_{w}(t)=V_{w}\left(t_{*}\right) / c_{\mathrm{ref}}, & x_{w 0}=X_{w 0} / X_{\mathrm{ref}}, & v_{w 0}=V_{w 0} / c_{\mathrm{ref}}, & F(t)=F_{*}\left(t_{*}\right) /\left(\rho_{0 *} c_{\mathrm{ref}}^{2}\right), \\
G(t)=G_{*}\left(t_{*}\right) /\left(\rho_{0 *} c_{\mathrm{ref}}^{2}\right), & M=M_{*} /\left(\rho_{0 *} X_{\mathrm{ref}}\right), & f\left(x_{i}, \zeta_{i}, t\right)=f_{*}\left(X_{i}, \xi_{i}, t_{*}\right) /\left(\rho_{0 *} / c_{\mathrm{ref}}^{3 / 2}\right),
\end{array}
$$

where $X_{\text {ref }}=\left(2 R T_{0 *}\right)^{1 / 2} / \omega_{*}$ is the reference length scale, $t_{\text {ref }}=1 / \omega_{*}$ is the reference time scale, $c_{\text {ref }}=\left(2 R T_{0 *}\right)^{1 / 2}$ is the reference speed, and $R$ is the specific gas constant (the Boltzmann constant divided by the molecular mass).

In the present one-dimensional problem, in which the physical quantities do not depend on $x_{2}$ and $x_{3}$, if we introduce the marginal velocity distribution function $g\left(x_{1}, \zeta_{1}, t\right)=\iint_{-\infty}^{\infty} f\left(x_{1}, \zeta_{i}, t\right) \mathrm{d} \zeta_{2} \mathrm{~d} \zeta_{3}$, then we can eliminate the components $\zeta_{2}$ and $\zeta_{3}$ of the molecular velocity from our problem. That is, the Boltzmann equation for the free-molecular gas (or the free-transport equation) reads

$$
\partial_{t} g+\zeta_{1} \partial_{x} g=0
$$

the corresponding initial condition is

$$
g=g_{0}, \quad g_{0}=\pi^{-1 / 2} \exp \left(-\zeta_{1}^{2}\right), \quad(t=0),
$$


and the diffuse-reflection boundary condition on the plate is written as

$$
\begin{aligned}
g\left(x_{1}, \zeta_{1}, t\right) & =g_{w \pm}\left(\zeta_{1}, t\right), \quad\left[x_{1}=x_{w \pm}(t), \zeta_{1}-v_{w}(t) \gtrless 0\right], \\
g_{w \pm}\left(\zeta_{1}, t\right) & =\pi^{-1 / 2} \rho_{w \pm}(t) \exp \left(-\left[\zeta_{1}-v_{w}(t)\right]^{2}\right), \\
\rho_{w \pm}(t) & =\mp 2 \sqrt{\pi} \int_{\zeta_{1}-v_{w}(t) \lessgtr 0}\left[\zeta_{1}-v_{w}(t)\right] g\left(x_{w \pm}(t), \zeta_{1}, t\right) \mathrm{d} \zeta_{1},
\end{aligned}
$$

where $x_{w+}\left(=x_{w}+0\right)$ and $x_{w-}\left(=x_{w}-0\right)$ stand for the surface of the plate facing to the positive $x_{1}$ direction and the negative $x_{1}$ direction, respectively. In Eq. (6) and in what follows, the upper (or lower) signs go together. The equation of the motion of the plate per unit area is written as

$$
\begin{gathered}
\mathrm{d} x_{w} / \mathrm{d} t=v_{w}(t), \quad M\left(\mathrm{~d} v_{w} / \mathrm{d} t\right)=F(t)-G(t), \\
F(t)=-M x_{w}(t), \quad G(t)=G_{+}(t)+G_{-}(t), \\
G_{ \pm}(t)= \pm\left(\int_{\zeta_{1}-v_{w}(t) \lessgtr 0}\left[\zeta_{1}-v_{w}(t)\right]^{2} g\left(x_{w \pm}(t), \zeta_{1}, t\right) \mathrm{d} \zeta_{1}+\int_{\zeta_{1}-v_{w}(t) \gtrless 0}\left[\zeta_{1}-v_{w}(t)\right]^{2} g_{w \pm}\left(\zeta_{1}, t\right) \mathrm{d} \zeta_{1}\right),
\end{gathered}
$$

and their initial conditions are given as

$$
x_{w}(0)=x_{w 0}, \quad v_{w}(0)=v_{w 0} .
$$

Here, $G_{ \pm}(t)$ are the drag acting on $x_{w \pm}(t)$. The dimensionless parameters characterizing the present problem are the mass ratio $M$, i.e., the density of the plate divided by the reference density of the gas, the initial position of the plate $x_{w 0}$, and the initial velocity of the plate $v_{w 0}$. For various sets of $M, x_{w 0}$, and $v_{w 0}$, we solve the coupled system, Eqs. (4) $-(6)$ and Eqs. (7) and (8) numerically, with special interest in the long-time behavior of the motion of the plate $x_{w}(t)$ and $v_{w}(t)$.

\section{INTEGRAL EQUATION FOR THE NUMERICAL ANALYSIS}

The equations for the gas, Eqs. (4)-(6), can be converted into the integral equations for $\rho_{w \pm}(t)$, which are more convenient for numerical analysis. We will give a brief outline of the derivation of the integral equations for the present one-dimensional problem (for the details, see [8], in which the corresponding integral equations are derived for two-dimensional case in the problem of unsteady motion of an infinite plate with finite width caused by a constant external force).

The free transport equation (4) indicates that the velocity distribution function $g\left(x_{1}, \zeta_{1}, t\right)$ is constant along the characteristics $x_{1}-\zeta_{1} t=$ const. In the following discussion, we fix the present time $t$ and introduce the time variable

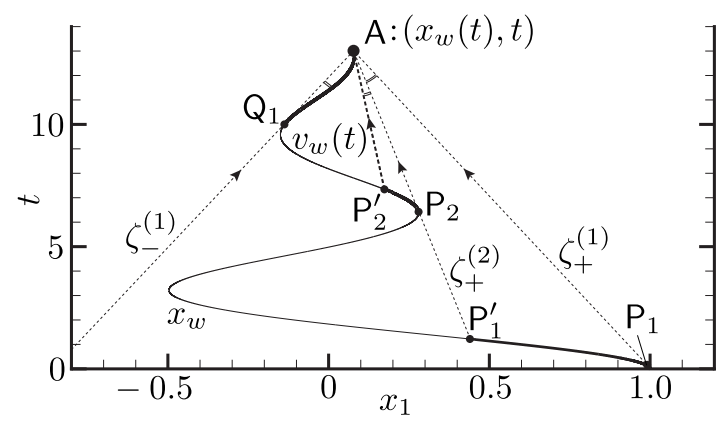

(a)

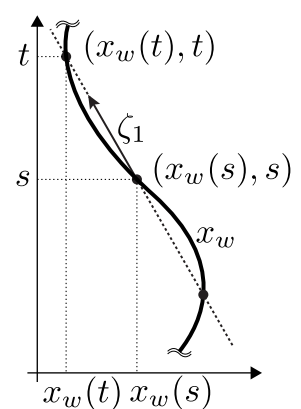

(b)

FIGURE 2. (a): The trajectory of the plate (the solid curve) and the segments that can be seen from a point $\mathrm{A}$ : $\left(x_{w}(t), t\right)($ the thick solid curve). The dashed lines drawn downward from the point $\mathrm{A}$ are tangent to the trajectory of the plate. From the point $\mathrm{A}_{+}$: $\left(x_{w+}(t), t\right)$ (the right-side face of the plate), the segment $\mathrm{P}_{1} \mathrm{P}_{1}^{\prime}$ and $\mathrm{P}_{2} \mathrm{P}_{2}^{\prime}$ can be seen, and from the point $\mathrm{A}_{-}:\left(x_{w-}(t), t\right)$ (the left side face of the plate) the segment $\mathrm{Q}_{1} \mathrm{~A}_{-}$can be seen. (b): The relation between $x_{w}(t), x_{w}(s), t, s\left[=s\left(\zeta_{1}, t\right)\right]$, and $\zeta_{1}$. The molecules that depart from $x_{w}(s)$ at time $s(<t)$ hit the plate $x_{w}(t)$ at time $t$ with the velocity $\zeta_{1}$ [cf. Eq. (10)]. 
in the past $s(s \in[0, t])$. Let us consider the gas molecules impinging on the plate surface $x_{1}=x_{w \pm}$ at time $t$. If we trace back the trajectories of such molecules, we either hit on the trajectory of the plate at a time in the past $s$ or reach the initial distribution $g_{0}$ without hitting on the trajectory. The former molecules correspond to the recolliding molecules. With these facts in mind, we can express the right-hand side of Eq. (6c) in terms of $g$ in the past by the following procedure.

The typical trajectory of the plate in $\left(x_{1}, t\right)$ plane under the elastic force is shown in Fig. 2(a). Figure 2(a) is in fact one of the trajectories $x_{1}=x_{w}(s), s \in[0, t]$ ( $t=13$ in the figure) computed from (4)-(8) numerically, where the parameters are chosen in such a way that the description of solution method is facilitated $\left(M=5, x_{w 0}=1\right.$, and $\left.v_{w 0}=0\right)$. Consider the molecules impinging on the plate at a point $\left(x_{w}(t), t\right)$, which is denoted by the symbol A in the figure. The solid curve indicates the trajectory of the plate $x_{1}=x_{w}(s), s \in[0, t]$, and the straight dashed lines, drawn downward from $\mathrm{A}$, show some of the trajectories of impinging molecules $x_{1}-\zeta_{1} s=x_{w}(t)-\zeta_{1} t$. The velocity of an impinging molecule is computed from the slope of its trajectory in $\left(x_{1}, t\right)$ space, and the faster the molecule, the milder the slope of the trajectory.

For brevity, we consider the molecules that hit the right-side surface $x_{1}=x_{w+}(t)$ (the plate surface facing to the positive $x_{1}$ direction). There are a finite number of segments of the trajectory $x_{1}=x_{w+}(s), s \in[0, t)$ that can be seen from point $A$ without being hidden by the trajectory of the plate itself. In the case of Fig. 2(a), the segment $P_{1} P_{1}^{\prime}$ and $\mathrm{P}_{2} \mathrm{P}_{2}^{\prime}$ are such segments. We denote the molecular velocities, corresponding to the tangential lines to the trajectory $x_{1}=x_{w}(s)$ drawn from A, by $\zeta_{+}^{(n)}$, where $\zeta_{+}^{\left(n_{1}\right)}<\zeta_{+}^{\left(n_{2}\right)}$ if $n_{1}<n_{2}$ and $n=1,2, \cdots, n_{+}\left[n_{+}=2\right.$ in the case of Fig. 2(a)]. Then, we can rewrite Eq. (6c) as

$$
-\frac{\rho_{w+}(t)}{2 \sqrt{\pi}}=\int_{-\infty}^{v_{w}(t)}\left[\zeta_{1}-v_{w}(t)\right] g_{0}\left(\zeta_{1}\right) \mathrm{d} \zeta_{1}+\sum_{n=1}^{n_{+}} \int_{\zeta_{+}^{(n)}}^{\zeta_{+}^{(n+1)}}\left[\zeta_{1}-v_{w}(t)\right]\left[g_{w}\left(\zeta_{1}, s\left(\zeta_{1}, t\right)\right)-g_{0}\left(\zeta_{1}\right)\right] \mathrm{d} \zeta_{1},
$$

where the time in the past $s\left(\zeta_{1}, t\right) \in[0, t)$ is obtained implicitly from the equation

$$
\frac{x_{w+}(t)-x_{w+}\left(s\left(\zeta_{1}, t\right)\right)}{t-s\left(\zeta_{1}, t\right)}=\zeta_{1}
$$

[see Fig. 2(b)] and $\sum_{n=1}^{n_{+}} \cdots=0$ if $n_{+}=0$. If $s\left(\zeta_{1}, t\right)$ obtained from (10) is multivalued as shown in Fig. 2(b), choose the largest $s \in[0, t)$. In addition, notice that $\zeta_{+}^{\left(n_{+}+1\right)}=v_{w}(t)$ holds in (9) [see Fig. 2(a)]. The first term on the right-hand side of (9) is the contribution if all the impinging molecules at time $t$ come from the initial velocity distribution $g_{0}$. The impinging molecules whose velocity $\zeta_{1}$ is contained in the ranges of integration in the terms under summation $\sum_{n=1}^{n_{+}}$ departed from the plate in the past at time $s\left(\zeta_{1}, t\right)$, not from the initial distribution. The correction caused by these molecules, which we call the recolliding molecules, is made by the terms under the summation $\sum_{n=1}^{n_{+}}$. In the same way, $G_{+}(t)$ in $(7 \mathrm{c})$ is recast as

$$
\begin{aligned}
G_{+}(t)= & \int_{-\infty}^{v_{w}(t)}\left[\zeta_{1}-v_{w}(t)\right]^{2} g_{0}\left(\zeta_{1}\right) \mathrm{d} \zeta_{1}+\sum_{n=1}^{n_{+}} \int_{\zeta_{+}^{(n)}}^{\zeta_{+}^{(n+1)}}\left[\zeta_{1}-v_{w}(t)\right]^{2}\left[g_{w}\left(\zeta_{1}, s\left(\zeta_{1}, t\right)\right)-g_{0}\left(\zeta_{1}\right)\right] \mathrm{d} \zeta_{1} \\
& +\int_{v_{w}(t)}^{\infty}\left[\zeta_{1}-v_{w}(t)\right]^{2} g_{w+}\left(\zeta_{1}, t\right) \mathrm{d} \zeta_{1} .
\end{aligned}
$$

The equations for $\rho_{w-}(t)$ and $G_{-}(t)$, corresponding to Eqs. (9) and (11), respectively, are readily obtained in the same way. Equations (9) and (10) and those for $\rho_{w-}(t)$, with explicit form of $g_{w \pm}$ [Eq. (6b)], are the integral equation for $\rho_{w+}(t)$ and $\rho_{w-}$ to be solved together with Eqs. (7a), (7b), (8), and (11) and the equation for $G_{-}(t)$. Since the numerical scheme is very similar to that used in [8], it is omitted here.

\section{NUMERICAL RESULT}

Before presenting the numerical results, we introduce the following auxiliary function $\alpha: \alpha(h)=\mathrm{d} \log |h| / \mathrm{d} \log t$, $\left[h=x_{w}(t)\right.$ or $\left.v_{w}(t)\right]$, where $\log (\cdot)$ is the common $\log \operatorname{arithm}\left[\log (\cdot) \equiv \log _{10}(\cdot)\right]$. Hence, if we have $\alpha(h) \approx p$, it indicates $h \approx t^{p}$. The numerical results of $\alpha\left(x_{w}\right)$ and $\alpha\left(v_{w}\right)$ are summarized in table 1 . Figure 3 shows the behavior of $x_{w}$ for cases 1, 4, 6, and 7 in table 1. To be more precise, Fig. 3(a) shows the single logarithmic plot of $x_{w}(t)$ vs $t$ for case 1 and case $1^{\prime}$ (see the next paragraph for case $1^{\prime}$ ). Figure 3(b) shows the double logarithmic plot of $x_{w}(t)$ vs $t$ for cases 1, 4, 6, and 7. Figure 3(c) shows the plot of $\alpha\left(x_{w}\right)$ vs $t$ for cases 1, 4, 6, and 7. 
TABLE 1. Values of $\alpha\left(x_{w}\right)$ and $\alpha\left(v_{w}\right)$ for different parameters $M, x_{w 0}$, and $v_{w 0}$ at $\log t=3.0,3.5$, and 4.0.

\begin{tabular}{c||rrr|rcc|ccc}
\hline \multicolumn{1}{l||}{} & \multicolumn{3}{c|}{ Parameters } & \multicolumn{3}{c|}{$-\alpha\left(x_{w}\right)$} & \multicolumn{3}{c}{$-\alpha\left(v_{w}\right)$} \\
\hline Case & $M$ & $x_{w 0}$ & $v_{w 0}$ & $\log t=3.0$ & $\log t=3.5$ & $\log t=4.0$ & $\log t=3.0$ & $\log t=3.5$ & $\log t=4.0$ \\
\hline $1^{*}$ & 10 & 1 & 0 & 1.99464 & 1.99833 & 1.99947 & 2.99189 & 2.99749 & 2.99921 \\
$2^{*}$ & 5 & 1 & 0 & 1.99994 & $\ldots$ & $\ldots$ & 2.99990 & $\ldots$ & $\ldots$ \\
$3^{*}$ & 2 & 1 & 0 & 2.00291 & $\ldots$ & $\ldots$ & 3.00436 & $\ldots$ & $\ldots$ \\
4 & 1 & 1 & 0 & 2.00446 & 2.00140 & 2.00044 & 3.00670 & 3.00211 & 3.00066 \\
$5^{*}$ & 10 & 0.5 & 0 & 1.99314 & $\ldots$ & $\ldots$ & 2.98962 & $\ldots$ & $\ldots$ \\
$6^{*}$ & 10 & 0.1 & 0 & 1.99250 & 1.99766 & 1.99926 & 2.98864 & 2.99649 & 2.99889 \\
7 & 1 & 0.1 & 0 & 2.00412 & 2.00130 & 2.00041 & 3.00619 & 3.00195 & 3.00061 \\
$8^{*}$ & 10 & 0 & 1 & 1.99585 & $\ldots$ & $\ldots$ & 2.99367 & $\ldots$ & $\ldots$ \\
$9^{*}$ & 10 & 0 & 0.1 & 1.99541 & $\ldots$ & $\ldots$ & 2.99301 & $\ldots$ & $\ldots$ \\
\hline
\end{tabular}

* case with oscillatory motion of the plate.

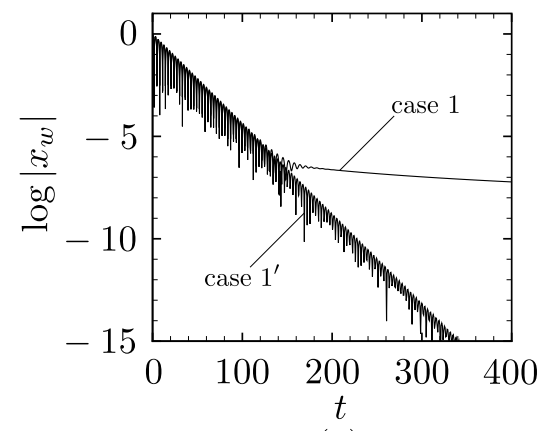

(a)

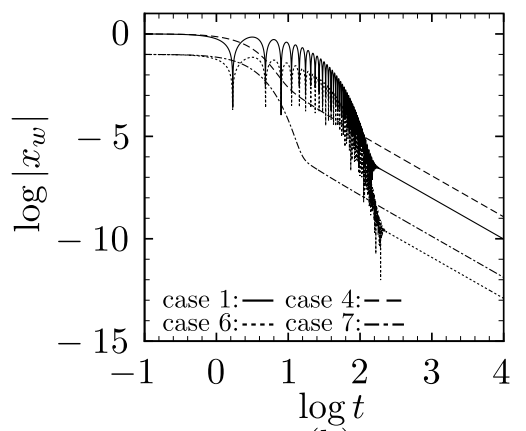

(b)

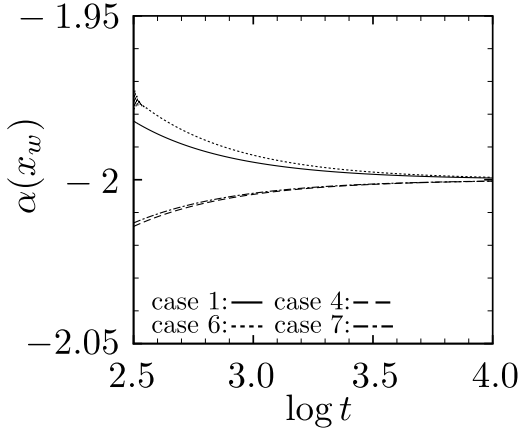

(c)

FIGURE 3. Long-time behavior of the position of the plate $x_{w}(t)$ for cases $1,4,6$, and 7. (a) $\log \left|x_{w}\right|$ vs $t$ for case 1 . In case $1^{\prime}$, recollision is neglected. (b) $\log \left|x_{w}\right|$ vs $\log t$. (c) $\alpha\left(x_{w}\right)$ vs $\log t$. In (b) and (c), the solid line indicates case 1 , the dotted line case 4 , the dashed line case 6 , and dash-dot line case 7.

In Fig. 3(a), case 1' indicates the result for the same parameters as case 1, but the effect of recollision is neglected, i.e., the terms under summation in Eqs. (9), (11) and the corresponding terms for $\rho_{w-}$ and $G_{-}$are neglected in the computation. The difference between case 1 and case $1^{\prime}$ is invisible until $t \simeq 150$. However, a completely different behavior is observed for the two cases after $t \simeq 150$. In the absence of the effect of recollision (case $1^{\prime}$ ), $x_{w}$ decays rapidly, oscillating around the equilibrium position $\left(x_{1}=0\right)$, in such a manner that the amplitude of oscillation approaches zero exponentially in $t$. In contrast, the result including the effect of recollision (case 1) demonstrates a slower decay of $\left|x_{w}\right|$. In addition, $\left|x_{w}\right|$ for case 1 decreases monotonically in $t$ for large $t$ without oscillation around $x_{1}=0$. It should also be noted that, at the beginning of bifurcation of the two cases after $t \simeq 150$, a tiny oscillation without crossing $x_{1}=0$ is observed for case 1 .

From Fig. 3(b), we observe that $\log \left|x_{w}\right|$ tends to approach a linearly decreasing function of $\log t$ for $t$ larger than $t \simeq 300(\log t \simeq 2.5)$ for all of cases 1, 4, 6, and 7. In fact, Fig. 3(c) and table 1 show that the gradient $\alpha\left(x_{w}\right)$ of each curve in Fig. 3(b) for large $t$ tends to approach -2 as $t$ increases. The values of $\alpha\left(x_{w}\right)$ for other cases in table 1 (cases $2,3,5,8$, and 9) also support this conclusion. Similarly, it is seen from the values of $\alpha\left(v_{w}\right)$ in table 1 that $\alpha\left(v_{w}\right)$ tends to approach -3 as time goes on (the figures for $v_{w}$ corresponding to Fig. 3 are omitted here because of limited space). In summary, we may conclude that the displacement of the plate and the velocity of its motion decay in the following manner for sufficiently large $t$ :

$$
\left|x_{w}\right| \approx C_{x} / t^{2}, \quad\left|v_{w}\right| \approx C_{v} / t^{3},
$$

where $C_{x}$ and $C_{v}$ are positive constants. It is natural that the velocity decays faster by $1 / t$ because $v_{w}=\mathrm{d} x_{w} / \mathrm{d} t$. It should be mentioned that cases $1,2,3,5,6,8$, and 9 demonstrate the oscillation of the plate around $x_{1}=0$. These oscillatory cases are beyond the mathematical proof in [5]. We should also note that diffuse reflection gives rise to a slower decay than specular reflection [cf. Eq. (2) with $d=1$ ].

Finally, we show the velocity distribution function for impinging molecules on the plate. Figure 4 shows $g\left(x_{1}, \zeta_{1}, t\right)$ $\left[x_{1}=x_{w \pm}(t), \zeta_{1} \lessgtr v_{w}(t)\right]$ for case 2 at (a) $t=4$, (b) $t=13$, and (c) $t=27$. The long-dashed line indicates $\zeta_{1}=v_{w}$. 


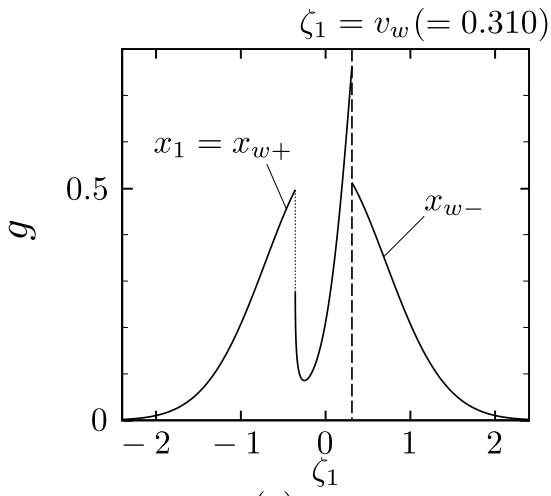

(a) $t=4$

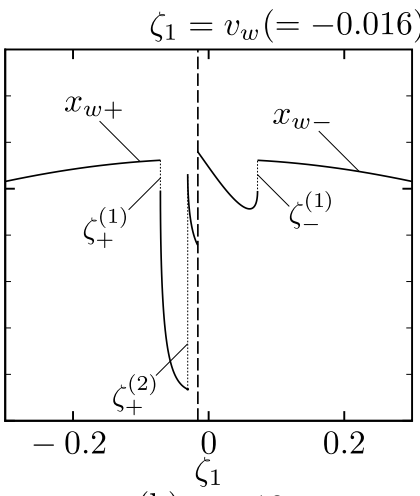

(b) $t=13$

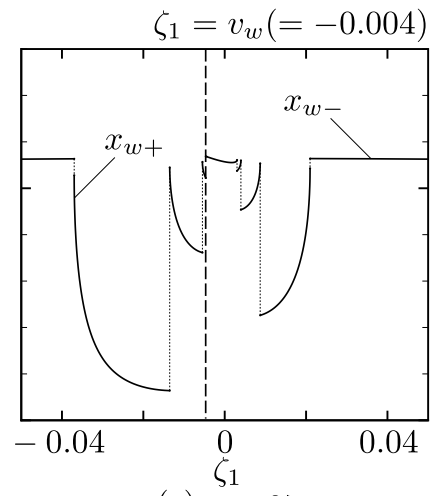

(c) $t=27$

FIGURE 4. The velocity distribution function for impinging molecules on the plate $: g\left(x_{1}, \zeta_{1}, t\right)\left[x_{1}=x_{w \pm}(t), \zeta_{1} \lessgtr v_{w}(t)\right]$ vs molecular velocity $\zeta_{1}$ for case 2 . (a) $t=4$, (b) $t=13$, and (c) $t=27$. The velocity $\zeta_{1}=v_{w}(t)$ is indicated by the long dashed line. Figure 4(b) corresponds to the situation of Fig. 2(a).

For $\zeta_{1}>v_{w}$ (or $\zeta_{1}<v_{w}$ ), the distribution function for molecules impinging on the left (or right) side is depicted. The velocity distribution function is, in general, discontinuous at $\zeta_{1}=\zeta_{ \pm}^{(n)}$ (the dotted line in the figure; see the section "Integral equation for numerical analysis" for $\zeta_{ \pm}^{(n)}$ ). Figure $4(\mathrm{~b})$ is the velocity distribution functions corresponding to Fig. 2(a). It is also seen from the figure that, at $\zeta_{1}=\zeta_{ \pm}^{(n)}$, the derivative of distribution function with respect to $\zeta_{1}$ diverges. These facts make the computation difficult generally. The use of the integral equations for $\rho_{w \pm}$ has the advantage of dealing with the discontinuities (or the singularities) accurately.

\section{CONCLUDING REMARKS}

In the present study, we have investigated the unsteady motion of an infinite plate in a free-molecular gas caused by an elastic force, with special interest in the decay rate of the motion, under the diffuse-reflection condition on the plate. The study complements the mathematical study [5], where a disk of dimension $d(d=1,2,3)$ is considered under the specular-reflection boundary condition. In [5], the slow decay proportional to an inverse power of time [Eq. (2)] was proved when the disk does not oscillate. The present results provide numerical evidence that the slow decay proved in [5] is also true, except for the fact that diffuse reflection gives a slower decay by $1 / t_{*}$ [cf. Eq. (2) with $d=1$ and Eq. (12)], when the motion of the plate is oscillatory. In addition, it is demonstrated that, even in the case of oscillatory motion, the motion decays monotonically without oscillation after sufficiently large times.

\section{ACKNOWLEDGMENTS}

The authors thank Prof. C. Marchioro, Prof. M. Pulvirenti, and Dr. G. Cavallaro for their valuable discussions.

\section{REFERENCES}

1. S. A. Schaaf and P. L. Chambré, in Fundamentals of Gas Dynamics, edited by H. W. Emmons, Princeton University Press, Princeton, 1958, Chap. H.

2. M. N. Kogan, Rarefied Gas Dynamics, Plenum, New York, 1969.

3. Y. Sone, Molecular Gas Dynamics: Theory, Techniques, and Applications, Birkäuser, Boston, 2007.

4. S. Caprino, C. Marchioro, and M. Pulvirenti, Commun. Math. Phys. 264, pp. 167-189 (2006).

5. S. Caprino, G. Cavallaro, and C. Marchioro, Math. Models. Meth. Appl. Sci. 17, pp. 1369-1403 (2007).

6. G. Cavallaro, Rendiconti di Matematica, Ser. VII 27, pp. 123-145 (2007).

7. K. Aoki, G. Cavallaro, C. Marchioro, and M. Pulvirenti, Math. Modell. Numer. Anal. 42, pp. 263-275 (2008).

8. K. Aoki, T. Tsuji, and G. Cavallaro, Phys. Rev. E 80, 016309 (2009).

9. G. Cavallaro and C. Marchioro, Math. Models. Meth. Appl. Sci. (to be published). 
Copyright of AIP Conference Proceedings is the property of American Institute of Physics and its content may not be copied or emailed to multiple sites or posted to a listserv without the copyright holder's express written permission. However, users may print, download, or email articles for individual use. 